\title{
Table of hyperfine anomaly in atomic systems
}

\author{
J.R. Persson \\ Department of Physics \\ NTNU \\ NO-7491 Trondheim \\ Norway \\ E-mail: jonas.persson@ntnu.no
}

October 30, 2018

\begin{abstract}
This table is a compilation of experimental values of magnetic hyperfine anomaly in atomic and ionic systems. The last extensive compilation was published in 1984 by Buttgenbach [Hyperfine Interactions 20 (1984) 1] and the aim here is to make an up to date compilation. The literature search covers the period to January 2011.
\end{abstract}




\section{Introduction}

The atomic electron-nuclear hyperfine interactions have been used to obtain nuclear spins and nuclear multipole moments [1, 2]. Isotopic shifts in spectral lines allows determination of the variation of the distribution of nuclear charge, essentially $\Delta\left\langle r^{2}\right\rangle$. Experiments on the magnetic counterpart, the distribution of nuclear magnetisation, are more difficult and only a few systematic measurements have been performed [4, 5, 3. The effect of an extended nuclear magnetisation is manifested by the difference between the point-like and actual magnetic dipole hyperfine interaction. This effect was first anticipated by Kopfermann [6] and thought to be too small to be observed. Following the experimental observation by Bitter [7] the effect of extended magnetisation was calculated by Bohr and Weisskopf [8] in 1950 and is therefore known as the Bohr-Weisskopf (B-W) effect. In order to probe the structural properties of the nucleus, the electron wave function must have a nonzero probability to be found at the origin. This means that only $s_{1 / 2}$ and relativistic $p_{1 / 2}$-electrons can be used. Note that electron-electron interaction can cause an s- or p- electron like behaviour, thus giving rise to an apparent nonzero probability for other electrons.

The magnetic dipole hyperfine interaction for the electron-nuclear system is represented by the Hamiltonian

$$
H=a I \cdot J,
$$

Where $a$ is the magnetic dipole hyperfine interaction constant. I and $\mathrm{J}$ are the nuclear and electron angular momenta. For an extended nucleus, the point-like hyperfine interaction constant $a_{\text {point }}$, is modified by two effects:

1. The modification of the electron wavefunctions by the extended nuclear charge distribution, the "Breit-Rosenthal-Crawford-Schawlow" correction $\left(\epsilon_{B R}\right)$ [9, 10, 11, 12,

2. The extended nuclear magnetisation, the Bohr-Weisskopf effect, $\left(\epsilon_{B W}\right)[8]$. We thus have

$$
a=a_{\text {point }}\left(1+\epsilon_{B W}\right)\left(1+\epsilon_{B R}\right)
$$

Where $a$ denotes the experimental value of the magnetic dipole hyperfine interaction constant. The hypothetical $a_{\text {point }}$ can not be calculated with sufficient precision for ordinary atoms, as is the case in muonic atoms and hydrogen-like ions. However, these uncertainties in point-like interactions cancel if we take the ratio of the $a$ values for two isotopes:

$$
\frac{a_{1}}{a_{2}}=\frac{g_{I}(1)}{g_{I}(2)} \frac{\left[1+\epsilon_{B W}(1)\right]\left[1+\epsilon_{B R}(1)\right]}{\left[1+\epsilon_{B W}(2)\right]\left[1+\epsilon_{B R}(2)\right]}
$$


Where $g_{I}=-\mu_{I} / I$ is the nuclear gyromagnetic ratio. As the $\epsilon$ are generally small, we get

$$
\begin{array}{r}
\frac{a_{1}}{a_{2}} \approx \frac{g_{I}(1)}{g_{I}(2)}\left[1+\epsilon_{B W}(1)-\right. \\
=\frac{\left.\epsilon_{B W}(2)\right]\left[1+\epsilon_{B R}(1)-\epsilon_{B R}(2)\right]}{g_{I}(2)}\left[1+{ }^{1} \Delta_{B W}^{2}\right]\left[1+{ }^{1} \Delta_{B R}^{2}\right]
\end{array}
$$

Where we use the definitions for the differential hyperfine anomaly of Bohr-Weisskopf and Breit-Rosenthal corrections, respectively

$$
\begin{gathered}
{ }^{1} \Delta_{B W}^{2} \equiv \epsilon_{B W}(1)-\epsilon_{B W}(2) \\
{ }^{1} \Delta_{B R}^{2} \equiv \epsilon_{B R}(1)-\epsilon_{B R}(2)
\end{gathered}
$$

Calculations of $\epsilon_{B R}$ show significant values, while the differential ${ }^{1} \Delta_{B R}^{2}$ is expected to be small and negligible compared to ${ }^{1} \Delta_{B W}^{2}[12$. However, in cases where the nuclei are very similar ${ }^{1} \Delta_{B R}^{2}$ will dominate. Dropping the subscripts we obtain

$$
\frac{a_{1}}{a_{2}} \approx \frac{g_{I}(1)}{g_{I}(2)}\left[1+{ }^{1} \Delta^{2}\right]
$$

Because the hyperfine anomaly is a quantity of the order of $10^{-3}$, it is necessary to know the hyperfine interaction constants, $a$, and the nuclear gyromagnetic values with at least an accuracy of $10^{-4}$ or better to obtain values accurate to $10 \%$ for the hyperfine anomaly [3]. Precision values of the hyperfine interaction constants, $a$, and independently measured gyromagnetic ratios are thus needed to obtain the differential hyperfine anomaly, ${ }_{1} \Delta^{2}$.

\section{State-dependent hyperfine anomaly and s-electron hyperfine anomaly}

The hyperfine anomaly shows a state dependence, where the values for different states can vary significantly, but shows an n-independence, as found in $\mathrm{Rb}$ [13. While the hyperfine anomalies normally are on the order of $1 \%$, state-dependent hyperfine anomaly can attain values up to $10 \%$.

The hyperfine interaction can be represented by the following operators 14 , 15]:

$$
h=\frac{\mu_{0}}{4 \pi} 2 \mu_{B} \sum_{i=1}^{N}\left[1\left\langle r^{-3}\right\rangle^{01}-\sqrt{10}\left(\mathrm{sC}^{2}\right)^{1}\left\langle r^{-3}\right\rangle^{12}+\mathrm{s}\left\langle r^{-3}\right\rangle^{10}\right]_{i} \cdot \mu_{I}
$$


where 1 and s are the orbital and spin angularmomentum operators, respectively, of the electron, $\mathrm{sC}^{2}$ is a tensor product of $\mathrm{s}$ and $\mathrm{C}^{2}$ of rank 1 . The indices stand for the rank in the spin and orbital spaces, respectively. Thus the hyperfine interaction can be considered to consist of three parts, orbital, spin-dipole and contact (spin) interaction, where only the contact (spin) interaction contributes to the hyperfine anomaly. This means that only s and $p_{1 / 2}$ electrons contribute to the hyperfine anomaly through the contact (spin) interaction. It is, therefore, suitable to rewrite the $a$ constant as

$$
a=a_{n c}+a_{c}
$$

Where $a_{c}$ is the contribution due to the contact interaction and $a_{n c}$ the contribution due to non-contact interactions. The experimental hyperfine anomaly, defined with the experimental $a$ constant, should then be rewritten to obtain the contact contribution to the hyperfine anomaly:

$$
{ }^{1} \Delta_{e x p}^{2}={ }^{1} \Delta_{c}^{2} \frac{a_{c}}{a}
$$

where ${ }^{1} \Delta_{c}^{2}$ is the hyperfine anomaly due to the contact interaction, that is, for an $s$ - or $p_{1 / 2}$-electron. The hyperfine anomaly is most often given as the state-dependent hyperfine anomaly, as the s-electron anomaly can be difficult to extract.

Using this result it is possible to extract the anomaly solely from the $a$-constants of two different atomic levels, provided the ratio $a_{c} / a$ differs substantially for the levels. Comparing the ratio of $a$-constants for two isotopes in two atomic levels, gives:

$$
\frac{a_{B}^{(1)} / a_{B}^{(2)}}{a_{C}^{(1)} / a_{C}^{(2)}} \approx 1+{ }^{1} \Delta_{c}^{2}\left(\frac{a_{c}^{B}}{a^{B}}-\frac{a_{c}^{C}}{a^{C}}\right)
$$

Where $\mathrm{B}$ and $\mathrm{C}$ denote different atomic levels and 1 and 2 denote different isotopes. The ratio between the two $a$-constant ratios for the isotopes will only depend on the difference of the contact contributions of the two atomic levels and the hyperfine anomaly for the s-electron. It should be pointed out that the atomic states used must differ significantly in the ratio $\frac{a_{c}}{a}$, as a small difference will lead to an increased sensitivity to errors [16]. Considering the special case where $\frac{a_{c}^{C}}{a^{C}}=0$, that is when the atomic level does not have any hyperfine anomaly, one can obtain values of the s-electron hyperfine anomaly for level B. This is common practice, however if the state does not exhibit any hyperfine anomaly the hyperfine structure is usually rather 
small and thus the relative error larger, leading to a large error for the hyperfine anomaly. The optimal case would be two atomic levels within the same multiplet where the experimental (or theoretical) $g_{J}$ is greater and smaller than 1, respectively. This is especially useful for unstable isotopes where there high precision measurements of the nuclear magnetic moment do not exist. Furthermore, states with a substantial difference in the ratio $a_{c} / a$ are also preferable for studies of the isotope shift. If measurements are performed on more than three atomic levels, it is also possible to deduce the nuclear magnetic moment ratio without the hyperfine anomaly. This will give the nuclear magnetic moment with high accuracy provided the nuclear magnetic moment is known for at least one stable isotope.

\section{Policies followed in the compilation}

The hyperfine anomaly is given with the lightest stable isotope as the reference isotope. The lightest naturally abundant isotope was used for $\mathrm{U}$ and the designation of the original article was used for Fr. In most cases the original article, where the hyperfine anomaly has been derived, is used. In the case of recent, more precise values of the nuclear magnetic moment, the hyperfine anomaly has been updated, accordingly. The nuclear magnetic moments of Stone [17] have been used, unless more precise values of ratios are available. Special care was taken to use magnetic moments obtained by the same method. The hyperfine anomaly is given as state-dependent if not stated otherwise. If the s-electron hyperfine anomaly is known, no extensive listing of state-dependent hyperfine anomaly is given, unless these are of special interest.

\section{References}

[1] E.W. Otten, Treatise on Heavy-Ion Science vol 8, ed. D. Allan Bromely (Plenum, New York 1989) 515

[2] J.Billowes and P. Campbell, J. Phys. G 21, (1995) 707.

[3] S. Büttgenbach, Hyperfine Int. 20, (1984) 1

[4] H.H. Stroke, V. Jaccarino, D.S. Edmonds, Jr and R. Weiss, Phys. Rev. 105, (1957) 590.

[5] P.A. Moskowitz, C.H. Liu, G. Fulop and H.H. Stroke, Phys. Rev. C4, (1971) 620 
[6] H. Kopfermann, Kernmomente (Akademische Verlagsgesellschaft, Leipzig, 1940)

[7] F. Bitter, Phys. Rev. 76,150 (1949)

[8] A. Bohr and V.F. Weisskopf, Phys. Rev. 77, (1950) 94

[9] E. Rosenthal and G. Breit, Phys. Rev. 41, (1932) 459

[10] M. Crawford and A. Schawlow, Phys. Rev. 76, (1949) 1310

[11] N.J. Ionesco-Pallas, Phys. Rev. 117, (1960) 505

[12] H.J. Rosenberg and H.H. Stroke, Phys. Rev. A 5, (1972) 1992

[13] Perez Galvan, A., Zhao, Y., Orozco, L.A., Gomez E., Lange, A.D., Baumer,A. and Sprouse, G.D.: Phys. Lett. 655B, 114 (2007)

[14] I. Lindgren and J. Morrison, Atomic Many-Body Theory (SpringerVerlag, Berlin 1983)

[15] P.G.H. Sandars and J. Beck, Proc. R. Soc. London A289, 97 (1965)

[16] J.R. Persson, Euro. Phys. J. A2,(1998) 3

[17] N.J. Stone At. Data Nucl. Data Tables 90 (2005) 75176 


\section{Table 1. Experimental data of hyperfine anomaly values in atomic systems}

\author{
Element \\ Isotope 1 \\ Isotope 2 \\ Atomic state/ s-anomaly \\ ${ }^{1} \Delta^{2}(\%)$ \\ Reference
}

The element studied

Reference isotope for the hyperfine anomaly

The second isotope used.

The atomic state for which the experimental hyperfine anomaly has been determined or the s-electron hfa. Hyperfine anomaly given in $\%$.

Original article where ${ }^{1} \Delta^{2}(\%)$ or the experimental hyperfine interactions constants is given. 
Table 1: Experimental data of hyperfine anomaly values in atomic systems

Element isotope 1 isotope 2 Atomic state/ s-anomaly

${ }^{1} \Delta^{2}(\%)$

Reference

$\mathrm{Li}$

6

7

$2 \mathrm{~s}{ }^{2} \mathrm{~S}_{1 / 2}$, s-anomaly

$3 \mathrm{~s}{ }^{2} \mathrm{~S}_{1 / 2}$, s-anomaly

$3 \mathrm{p}^{2} \mathrm{P}_{1 / 2}$

$\mathrm{N}$

$\mathrm{Na} \quad 23 \quad 24$

$\begin{array}{lll}\mathrm{Cl} & 35 & 37\end{array}$

$\begin{array}{lll}\text { K } & 39 & 37\end{array}$

$39 \quad 40$

$39 \quad 41$

$39 \quad 42$

$\begin{array}{lll}\mathrm{V} & 50 \quad 51\end{array}$

$\mathrm{Cu} \quad 63$

65

$$
51
$$

$3 d^{9} 4 s 4 p{ }^{4} \mathrm{P}_{5 / 2}$

$3 \mathrm{~d}^{9} 4 \mathrm{~s} 4 \mathrm{p}{ }^{4} \mathrm{P}_{9 / 2}$

Ga $\quad 69$

$69 \quad 71$

71

72

$\mathrm{Br}$

$\mathrm{Br} \quad 79$

$\mathrm{Rb}$

$$
79
$$

$$
85
$$

$$
85
$$$$
85
$$

84
86
87

81

84

86

87

$$
\begin{aligned}
& 4 \mathrm{p}{ }^{2} \mathrm{P}_{1 / 2} \\
& 4 \mathrm{p}{ }^{2} \mathrm{P}_{3 / 2} \\
& 4 \mathrm{p}{ }^{2} \mathrm{P}_{1 / 2} \\
& 4 \mathrm{p}{ }^{2} \mathrm{P}_{3 / 2} \\
& 4 \mathrm{p}{ }^{2} \mathrm{P}_{1 / 2} \\
& 4 \mathrm{p}{ }^{2} \mathrm{P}_{3 / 2} \\
& 4 \mathrm{p}^{5}{ }^{2} \mathrm{P}_{3 / 2} \\
& 5 \mathrm{~s}{ }^{2} \mathrm{~S}_{1 / 2}, \text { s-anomaly } \\
& 5 \mathrm{~s}{ }^{2} \mathrm{~S}_{1 / 2}, \text { s-anomaly } \\
& 5 \mathrm{~s}{ }^{2} \mathrm{~S}_{1 / 2}, \text { s-anomaly } \\
& 6 \mathrm{~s}{ }^{2} \mathrm{~S}_{1 / 2}, \text { s-anomaly } \\
& 7 \mathrm{~s}{ }^{2} \mathrm{~S}_{1 / 2}, \text { s-anomaly } \\
& 5 \mathrm{p}{ }^{2} \mathrm{P}_{1 / 2} \\
& 5 \mathrm{p}{ }^{2} \mathrm{P}_{3 / 2}
\end{aligned}
$$

$\begin{array}{lccc}\text { Mo } & 95 & 97 & 4 \mathrm{~d}^{5} 5 \mathrm{~s}^{7} \mathrm{~S}_{3} \\ \mathrm{Ru} & 99 & 101 & \text { s-anomaly } \\ \mathrm{Ag} & 107 & 103 & 4 \mathrm{~d}^{10} 5 \mathrm{~s}{ }^{2} \mathrm{~S}_{1 / 2}\end{array}$

$0.00681(7)$

$0.022(55)$

$-0.19(4)$

0.0999(4)

$0.0013(30)$

$-0.00381(2)$

$-0.249(35)$

$0.466(19)$

$-0.22936(14)$

$0.336(38)$

$0.0007(10)$

0.034(60)

$0.004861(9)$

$0.00340(11)$

$0.00305(17)$

$-0.00050(7)$

$0.00200(16)$

$0.00063(6)$

$-0.00252(12)$

$0.0043(6)$

$-0.0170(18)$

$-0.00003(4)$

$-1.7(1.0)$

$0.17(9)$

$0.35142(30)$

$0.36(2)$

$0.32(2)$

$0.673(7)$

$0.164(8)$

$-0.0101(2)$

$-0.0173(1)$

$-3.4(1.7)$
15] 16

24]

25]

14

14

14

14

14

17.

1920

21] 22

26 . 
Table 1: (continued)

Element isotope 1 isotope 2 Atomic state/ s-anomaly

$$
{ }^{1} \Delta^{2}(\%)
$$

Reference

$\begin{array}{lll}107 & 108 & 4 \mathrm{~d}^{10} 5 \mathrm{~s}{ }^{2} \mathrm{~S}_{1 / 2} \\ 107 & 109 & 4 \mathrm{~d}^{10} 5 \mathrm{~s}{ }^{2} \mathrm{~S}_{1 / 2} \\ 107 & 109^{m} & 4 \mathrm{~d}^{10} 5 \mathrm{~s}{ }^{2} \mathrm{~S}_{1 / 2} \\ 107 & 110 & 4 \mathrm{~d}^{10} 5 \mathrm{~s}{ }^{2} \mathrm{~S}_{1 / 2} \\ 107 & 110^{m} & 4 \mathrm{~d}^{10} 5 \mathrm{~s}{ }^{2} \mathrm{~S}_{1 / 2}\end{array}$

\begin{tabular}{cr}
$-2.6(7)$ & 27 \\
$-0.41274(29)$ & 28 \\
$-3.8(4.1)$ & 29 \\
$-0.85(1.19)$ & $29, \mu_{I}$ from $[30$ \\
$-3.1(1.4)$ & 27 \\
$-2.88(13)$ & 31 \\
\hline
\end{tabular}

$\mathrm{Cd}$

$\begin{array}{lll}111 & 107 & 5 \mathrm{~s} 5 \mathrm{p}{ }^{3} \mathrm{P}_{1} \\ 111 & 109 & 5 \mathrm{~s} 5 \mathrm{p}{ }^{3} \mathrm{P}_{1} \\ 111 & 113 & 5 \mathrm{~s} 5 \mathrm{p}{ }^{3} \mathrm{P}_{1} \\ 111 & 113 & 5 \mathrm{~s} 5 \mathrm{p}{ }^{3} \mathrm{P}_{2} \\ 111 & 113 & 5 \mathrm{~s} 6 \mathrm{~s}{ }^{3} \mathrm{~S}_{1} \\ 111 & 113^{m} & 5 \mathrm{~s} 5 \mathrm{p}{ }^{3} \mathrm{P}_{1} \\ 111 & 115 & 5 \mathrm{~s} 5 \mathrm{p}{ }^{3} \mathrm{P}_{1} \\ 111 & 115^{m} & 5 \mathrm{~s} 5{ }^{3} \mathrm{P}_{1}\end{array}$

\begin{tabular}{cc}
$-0.0958(8)$ & 32 \\
$-0.0912(7)$ & 32 \\
$-0.00023(40)$ & 33 \\
$-0.00143(6)$ & 34 \\
$-0.01(4)$ & 35 \\
$-0.0773(5)$ & 33 \\
$0.244(65)$ & 33 \\
$-0.236(90)$ & 33 \\
\hline
\end{tabular}

In $\quad 113 \quad 115 \quad 5 \mathrm{p}^{2} \mathrm{P}_{1 / 2}$

$5 \mathrm{p}^{2} \mathrm{P}_{3 / 2}$

$0.00075(13)$

36

$-0.00238(13)$

36

$\mathrm{Sn}$

115

$117 \quad 5 \mathrm{p}^{2}{ }^{3} \mathrm{P}_{1}$

$5 \mathrm{p}^{2}{ }^{3} \mathrm{P}_{2}$

$117 \quad 119 \quad 5 \mathrm{p}^{2}{ }^{3} \mathrm{P}_{1}$

$5 \mathrm{p}^{2}{ }^{3} \mathrm{P}_{2}$

$5 \mathrm{p}^{2}{ }^{1} \mathrm{D}_{2}$

$0,0034(10)$

$-0.0003(10) \quad 37$

$0.0049(10)$

$-0.0009(10) \quad 37$

$+0.0001(10) \quad 38$

$\mathrm{Sb} \quad 121 \quad 123 \quad 5 \mathrm{p}^{3}{ }^{4} \mathrm{~S}_{3 / 2}$

$-0.323(9)$

39

Cs $\quad 133 \quad 131 \quad 6 \mathrm{~s}^{2} \mathrm{~S}_{1 / 2}$, s-anomaly

$6 \mathrm{~s}^{2} \mathrm{~S}_{1 / 2}$, s-anomaly

$\begin{array}{ll}134^{m} & 6 \mathrm{~s}{ }^{2} \mathrm{~S}_{1 / 2}, \text { s-anomaly } \\ 135 & 6 \mathrm{~s}{ }^{2} \mathrm{~S}_{1 / 2}, \text { s-anomaly }\end{array}$

137

$6 s{ }^{2} \mathrm{~S}_{1 / 2}$, s-anomaly

$0.48(5)$

$0.17(3)$

$-1.38(3)$

40

133

133

137

$5 \mathrm{~d} 6 \mathrm{~s}{ }^{3} \mathrm{D}_{1}$

$5 \mathrm{~d} 6 \mathrm{~s}{ }^{3} \mathrm{D}_{2}$

$5 \mathrm{~d} 6 \mathrm{~s}{ }^{3} \mathrm{D}_{3}$

$5 \mathrm{~d} 6 \mathrm{~s}{ }^{1} \mathrm{D}_{2}$

$\mathrm{Ba}^{+} 6 \mathrm{~s}^{2} \mathrm{~S}_{1 / 2}$, s-anomaly

$0.04(1)$
$0.0018(40)$

41

41

$-0.205(7)$

$-0.179(22)$

$-0.188(17) 43$

$-0.212(26)$

$-0.191(5) \quad 45$

$\begin{array}{lrrrrr}\text { La } & 138 & 139 & 5 \mathrm{~d} 6 \mathrm{p}^{3} \mathrm{D}_{1} & -0.35(23) & \text { 46] 47] } \\ \mathrm{Nd} & 143 & 145 & \text { s-anomaly } & 0.2034(63) & 48 \text {. } \\ \mathrm{Eu} & 151 & 145 & \text { s-anomaly } & -0.08(15)\end{array}$


Table 1: (continued)

Element isotope 1 isotope 2 Atomic state/ s-anomaly

\begin{tabular}{|c|c|c|c|c|c|}
\hline & 151 & 146 & s-anomaly & $0.12(50)$ & \\
\hline & 151 & 147 & s-anomaly & $-0.12(17)$ & \\
\hline & 151 & 148 & s-anomaly & $0.08(31)$ & \\
\hline & 151 & 149 & s-anomaly & $-0.19(16)$ & \\
\hline & 151 & 150 & s-anomaly & $0.08(28)$ & \\
\hline & 151 & 152 & s-anomaly & $0.50(6)$ & \\
\hline & 151 & 153 & s-anomaly & $-0.64(3)$ & \\
\hline $\mathrm{Gd}$ & 155 & 157 & s-anomaly & $0.106(24)$ & \\
\hline Dy & 161 & 163 & $\begin{array}{l}4 \mathrm{f}^{10} 6 \mathrm{~s} 6 \mathrm{p}{ }^{5} \mathrm{~K}_{8} \\
4 \mathrm{f}^{10} 6 \mathrm{~s} 6 \mathrm{p}{ }^{5} \mathrm{~K}_{9} \\
4 \mathrm{f}^{10} 6 \mathrm{~s} 6 \mathrm{p}{ }^{5} \mathrm{I}_{8} \\
4 \mathrm{f}^{10} 6 \mathrm{~s} 6 \mathrm{p}{ }^{5} \mathrm{H}_{7}\end{array}$ & $\begin{array}{l}0.019(16) \\
0.025(11) \\
-0.116(19) \\
-0.176(36)\end{array}$ & \\
\hline $\mathrm{Yb}$ & 171 & 173 & $\begin{array}{l}6 \mathrm{~s} 6 \mathrm{p}{ }^{3} \mathrm{P}_{1} \\
4 \mathrm{f}^{13} 5 \mathrm{~d} 6 \mathrm{~s}^{2}{ }^{3} \mathrm{P}_{1} \\
\mathrm{Yb}^{+} 6 \mathrm{~s}^{2} \mathrm{~S}_{1 / 2}, \text { s-anomaly }\end{array}$ & $\begin{array}{l}-0.386(5) \\
0.066(22) \\
-0.425(5)\end{array}$ & 53 \\
\hline $\mathrm{Lu}$ & $\begin{array}{l}175 \\
176\end{array}$ & $\begin{array}{l}177 \\
176^{m}\end{array}$ & $\begin{array}{l}5 \mathrm{~d} 6 \mathrm{~s}^{2}{ }^{2} \mathrm{D}_{3 / 2} \\
5 \mathrm{~d} 6 \mathrm{~s}^{2}{ }^{2} \mathrm{D}_{5 / 2} \\
5 \mathrm{~d} 6 \mathrm{~s} 6 \mathrm{p}{ }^{4} \mathrm{P}_{1 / 2} \\
5 \mathrm{~d} 6 \mathrm{~s} 6 \mathrm{p}{ }^{4} \mathrm{P}_{3 / 2} \\
5 \mathrm{~d} 6 \mathrm{~s} 6 \mathrm{p}{ }^{4} \mathrm{P}_{5 / 2} \\
5 \mathrm{~d} 6 \mathrm{~s} 6 \mathrm{p}{ }^{4} \mathrm{~F}_{3,5,7 / 2}, \text { s-anomaly } \\
6 \mathrm{~s}^{2} 8 \mathrm{p}{ }^{2} \mathrm{P}_{1 / 2} \\
6 \mathrm{~s}^{2} 8 \mathrm{p}{ }^{2} \mathrm{P}_{3 / 2} \\
\text { s-anomaly } \\
\text { s-anomaly }\end{array}$ & $\begin{array}{c}0.02(15) \\
0.19(15) \\
0.40(24) \\
1.62(25) \\
0.0(27) \\
0.48(8) \\
1.84(90) \\
0.55(22) \\
-0.018(35) \\
0.48(8)\end{array}$ & 57, $\mu_{I}$ from \\
\hline $\mathrm{Re}$ & $\begin{array}{l}185 \\
185 \\
185 \\
185\end{array}$ & $\begin{array}{l}186 \\
187 \\
187 \\
188\end{array}$ & $\begin{array}{l}5 \mathrm{~d}^{5} 6 \mathrm{~s}^{2}{ }^{6} \mathrm{~S}_{5 / 2} \\
5 \mathrm{~d}^{5} 6 \mathrm{~s}^{2}{ }^{6} \mathrm{~S}_{5 / 2} \\
\mathrm{~s}-\text { anomaly } \\
5 \mathrm{~d}^{5} 6 \mathrm{~s}^{2}{ }^{6} \mathrm{~S}_{5 / 2}\end{array}$ & $\begin{array}{c}-1.36(17) \\
0.031(8) \\
0.027(5) \\
-1.28(28)\end{array}$ & 58 \\
\hline Ir & 191 & 193 & s-anomaly & $-0.64(7)$ & \\
\hline $\mathrm{Au}$ & $\begin{array}{l}197 \\
197 \\
197\end{array}$ & $\begin{array}{l}196 \\
198 \\
199\end{array}$ & $\begin{array}{l}\text { 6s }{ }^{2} \mathrm{~S}_{1 / 2}, \text { s-anomaly } \\
\text { 6s }{ }^{2} \mathrm{~S}_{1 / 2}, \text { s-anomaly } \\
6 s{ }^{2} \mathrm{~S}_{1 / 2}, \text { s-anomaly }\end{array}$ & $\begin{array}{l}8.69(26) \\
8.53(8) \\
3.64(29)\end{array}$ & $\begin{array}{r}62 \\
64 \\
64 \\
6\end{array}$ \\
\hline $\mathrm{Hg}$ & $\begin{array}{l}199 \\
199 \\
199\end{array}$ & $\begin{array}{l}193 \\
193^{m} \\
195\end{array}$ & $\begin{array}{l}6 \mathrm{~s} 6 \mathrm{p}{ }^{3} \mathrm{P}_{1} \\
6 \mathrm{~s} 6 \mathrm{p}{ }^{3} \mathrm{P}_{1} \\
6 \mathrm{~s} 6 \mathrm{p}{ }^{3} \mathrm{P}_{1}\end{array}$ & $\begin{array}{c}-0.61(3) \\
-1.0552(13) \\
-0.1470(9)\end{array}$ & \\
\hline
\end{tabular}


Table 1: (continued)

Element isotope 1 isotope 2 Atomic state/ s-anomaly

$\begin{array}{cr}{ }^{1} \Delta^{2}(\%) & \text { Reference } \\ -1.038(3) & 65 \\ -0.0778(7) & 65 \\ -1.021(3) & 65 \\ -0.960(9) & 65 \\ -0.1467(6) & 65 \\ -0.15653(4) & 65 \\ -0.16257(5) & 66 \\ -0.796(16) & 65 \\ 0.01035(15) & 67 \\ -0.16258(10) & 68\end{array}$

$195^{m} \quad 6 \mathrm{~s} 6 \mathrm{p}^{3} \mathrm{P}_{1}$

$6 \mathrm{~s} 6 \mathrm{p}{ }^{3} \mathrm{P}_{1}$

$6 \mathrm{~s} 6 \mathrm{p}{ }^{3} \mathrm{P}_{1}$

$6 \mathrm{~s} 6 \mathrm{p}{ }^{3} \mathrm{P}_{1}$

$6 \mathrm{~s} 6 \mathrm{p}^{3} \mathrm{P}_{1}$

$6 \mathrm{~s} 6 \mathrm{p}{ }^{3} \mathrm{P}_{2}$

$\mathrm{Hg}^{+}, 6 \mathrm{~s}^{2} \mathrm{~S}_{1 / 2}$, s-anomaly

$6 \mathrm{~s} 6 \mathrm{p}{ }^{3} \mathrm{P}_{1}$

$\begin{array}{lll}\mathrm{Tl} & 203 & 6 \mathrm{p}^{2} \mathrm{P}_{1 / 2} \\ 6 \mathrm{p}^{2} \mathrm{P}_{3 / 2}\end{array}$

$-0.032(38)$

69

$212 \quad 209$

$7 \mathrm{~s}^{2} \mathrm{~S}_{1 / 2}-7 \mathrm{p}^{2} \mathrm{P}_{1 / 2}\left(\Delta_{s}-\Delta_{p}\right)$

$0.339(31)$

69

$212 \quad 210$

$7 \mathrm{~s}^{2} \mathrm{~S}_{1 / 2}-7 \mathrm{p}^{2} \mathrm{P}_{1 / 2}\left(\Delta_{s}-\Delta_{p}\right)$

$\begin{array}{lr}7 \mathrm{~s}{ }^{2} \mathrm{~S}_{1 / 2}-7 \mathrm{p}{ }^{2} \mathrm{P}_{1 / 2}\left(\Delta_{s}-\Delta_{p}\right) & -0.007(28) \\ 7 \mathrm{~s}{ }^{2} \mathrm{~S}_{1 / 2}-7 \mathrm{p}{ }^{2} \mathrm{P}_{1 / 2}\left(\Delta_{s}-\Delta_{p}\right) & 0.331(34)\end{array}$

69

$212 \quad 211$

$5 \mathrm{f}^{3} 6 \mathrm{~d} 7 \mathrm{~s}^{2}{ }^{5} \mathrm{~L}_{6}$

$0.84(31)$

69 .

U $\quad 233$

235

$5 \mathrm{f}^{3} 6 \mathrm{~d} 7 \mathrm{~s} 7 \mathrm{p}{ }^{7} \mathrm{M}_{7}$

$5 f^{3} 6 \mathrm{~d} 7 \mathrm{~s} 7 \mathrm{p}{ }^{7} \mathrm{~L}_{6}$

$1.32(31)$

70

70

70 


\section{References}

[1] A. Beckmann, K.D. Boklen and D. Elke Z. Phys., 270(3):173-186, 1974.

[2] B.A. Bushaw, W. Nortershauser, G. Ewald, A. Dax and G.W.F. Drake Phys. Rev. Lett., 91(4), JUL 252003.

[3] D. Das and V. Natarajan J. Phys. B, 41(3), FEB 142008.

[4] W.W. HOlloway, R. Novick, and E. Luscher. Phys. Rev., 126(6):2109-\&, 1962.

[5] Y.W. Chan, V.W. Cohen, M. Lipsicas and H.B. Silsbee Phys. Rev., 150(3):933-\&, 1966.

[6] J.H. King, J.H. Holloway and B.B. Aubrey Hyperfine structure and nuclear magnetic octopole moment of the stable chlorine isotopes. Technical Report NP-6012, Massachusetts institute of Technology, 1956. Quarterly progress report, Research laboratory of electronics.

[7] C.V. Platen, J. Bonn, U. Kopf, R. Neugart and E.W. Otten Z. Phys., 244(1):44-\&, 1971.

[8] J.T. Eisinger, B. Bederson and B.T. Feld Phys. Rev., 86(1):73-81, 1952.

[9] Y.W. Chan, V.W. Cohen and H.B. Silsbee Phys. Rev., 184(4):1102-\&, 1969.

[10] O. Lutz, W. Messner, K.R. Mohn and P. Kroneck Z. Phys. A, 300(2-3):111-114, 1981.

[11] E.C.A Cochrane, D.M. Benton, D.H. Forest and J.A.R. Griffith J. Phys. B, 31(10):2203-2213, MAY 281998.

[12] O. Lutz, H. Oehler and P. Kroneck Z. Phys. A, 288(1):17-21, 1978.

[13] A.G. Blachman, D.A. Landman and A. Lurio Phys. Rev., 181(1):70-\&, 1969.

[14] O. Lutz, A. Nolle and A. Uhl Z. Phys., 248(2):159-\&, 1971.

[15] H.H. Brown and J.G. King Phys. Rev., 142(1):53-\&, 1966.

[16] O. Lutz Phys. Lett. A, A 31(7):384-\&, 1970.

[17] F. Ackermann, I. Platz and G. zu Pulitz Z. Phys., 260(2):87-110, 1973.

[18] N. Braslau, G.O. Brink and J.M. Khan Phys. Rev., 123(5):1801-\&, 1961.

[19] B. Bederson and V. Jaccarino Phys. Rev., 87(1):228-229, 1952.

[20] E. Arimondo, M. Inguscio and P. Violino Rev. Mod. Phys., 49(1):31-75, Jan 1977.

[21] A. Perez Galvan, Y. Zhao, L.A. Orozco, E. Gomez, A.D. Lange, F. Baumer and G.D. Sprouse Phys. Lett. B, 655(3-4):114-118, NOV 12007.

[22] A. Perez Galvan, Y. Zhao and L.A. Orozco Phys. Rev. A, 78(1), JUL 2008.

[23] H.C. Chui, M.S. Ko, Y.W. Liu, J.T. Shy, J.L. Peng and H. Ahn Opt. Lett., 30(8):842844, APR 152005.

[24] S. Buttgenbach, M. Herschel, G. Meisel, E. Schrodl, W. Witte and W.J. Childs Z. Phys., 266(4):271-274, 1974.

[25] S. Buttgenbach Hyperfine Int., 20(1):1-64, 1984.

[26] B. Wannberg, J.O. Jonsson, and L. Sanner Phys. Scr., 1(5-6):238-242, 1970.

[27] C.J. Cussens, G.K. Rochester and K.F. Smith J. Phys. A , 2(6):658-\&, 1969. 
[28] H. Dahmen and S. Penselin Z. Phys., 200(4):456-\&, 1967.

[29] G.M. Stinson, A.R. Pierce, J.C. Waddington and R.G. Summersgate Can. J. Phys., 49(7):906-\&, 1971.

[30] R. Eder, E. Hagn and E. Zech Phys. Rev. C, 31(1):190-196, 1985.

[31] S.G. Schmelling, V.J. Ehlers and H.A. Shugart Phys. Rev., 154(4):1142-\&, 1967.

[32] P. Thaddeus and M.N. Mcdermott Phys. Rev., 132(3):1186-\&, 1963.

[33] R.L. Chaney and M.N. Mcdermott Phys. Lett. A, A 29(2):103-\&, 1969.

[34] W. Faust, M. Mcdermott and W. Lichten Phys. Rev., 120(2):469, 1960.

[35] M. Chantepi Comptes Rendus Hebdomadaires Des Seances De L ACademie Des Sciences Serie B, 269(12):522-\&, 1969.

[36] T.G. Eck, A. Lurio and P. Kusch Phys. Rev., 106(5):954-957, 1957.

[37] W.J. Childs and L.S. Goodman Phys. Rev., 137(1A):A35-\&, 1965.

[38] W.J. Childs Phys. Rev. A, 4(2):439-\&, 1971.

[39] P.C.B Fernando, G.K. Rochester, I.J. Spalding and K.F. Smith Phil. Mag., 5(60):1291-1298, 1960.

[40] R.D. Worley, V.J. Ehlers, W.A. Nierenberg and H.A. Shugart Phys. Rev., 140(6B):1483-\&, 1965.

[41] H.H. Stroke, V. Jaccarino, D.S. Edmonds and R. Weiss Phys. Rev., 105(2):590-603, 1957.

[42] V.W. Cohen, T. Moran and S. Penselin Phys. Rev., 127(2):517-\&, 1962.

[43] M. Gustavsson, G. Olsson and A. Rosen Z. Phys. A, 290(3):231-243, 1979.

[44] S.G. Schmelling Phys. Rev. A, 9(3):1097-1102, 1974.

[45] S. Trapp, G. Marx, G. Tommaseo, A. Klaas, A. Drakoudis, G. Revalde, G. Szawiola and G. Werth hyperfine int., 127(1-4):57-64, 2000.

[46] H. Iimura, M. Koizumi, M. Miyabe, M. Oba, T. Shibata, N. Shinohara, Y. Ishida, T. Horiguchi and H.A. Schuessler Phys. Rev. C, 68(5), NOV 2003.

[47] W.J. Childs and L.S. Goodman Phys. Rev. A, 20(5):1922-1926, 1979.

[48] J.R. Persson arXiv:0904.0516 2009.

[49] J.R. Persson arXiv:0904.4828 2009.

[50] H. Brand, V. Pfeufer, and A. Steudel Z. Phys. A, 302(4):291-298, 1981.

[51] D.L. Clark and G.W. Greenlees Phys. Rev. C, 26(4):1636-1648, 1982.

[52] B. Budick and J. Snir Phys. Rev. A, 1(3):545-\&, 1970.

[53] P.T.H Fisk, M.J. Sellars, M.A. Lawn and C. Coles IEEE Transactions on Ultrasonics Ferroelectrics and Frequency Control, 44(2):344-354, MAR 1997.

[54] A. Munch, M. Berkler, C. Gerz, D. Wilsdorf and G. Werth Phys. Rev. A, 35(10):41474150, MAY 151987.

[55] T. Brenner, S. Buttgenbach, W. Rupprecht and F. Traber NucL. Phys. A, 440(3):407423, 1985.

[56] S. Witte, EJ. van Duijn, R. Zinkstok and W. Hogervorst Eur. Phys. J. D, 20(2):159164, AUG 2002. 
[57] A. Kuhnert, A. Nunnemann and D. Zimmermann J. Phys. B, 16(23):4299-4303, 1983.

[58] L. Armstrong and R. Marrus Phys. Rev., 138(2B):B310-\&, 1965.

[59] S. Buttgenbach, R. Dicke, G. Golz and F. Traber Z. Phys. A, 302(4):281-290, 1981.

[60] K.H. Burger, B. Burghardt, S. Buttgenbach, R. Harzer, H. Hoeffgen, G. Meisel and F. Traber Z. Phys. A, 307(3):201-209, 1982.

[61] S. Buttgenbach, R. Dicke, H. Gebauer, R. Kuhnen and F. Traber Z. Phys. A, 286(4):333-340, 1978.

[62] S.G. Schmelling, V.J. Ehlers and H.A. Shugart Phys. Rev. C, 2(1):225-\&, 1970.

[63] C. Ekstrom, L. Robertsson, S. Ingelman, G. Wannberg and I. Ragnarsson Nucl. Phys. A, 348(1):25-44, 1980.

[64] P.A. van den Bout., V.J. Ehlers, W.A. Nierenberg and H.A. Shugart Phys. Rev., 158(4):1078-\&, 1967.

[65] R.J. Reimann and M.N. Mcdermott Phys. Rev. C, 7(5):2065-2079, 1973.

[66] E.A. Burt, S. Taghavi-Larigani, and R.L. Tjoelker Phys. Rev. A, 79(6), JUN 2009.

[67] A. Lurio and A.G. Prodell Phys. Rev., 101(1):79-83, 1956.

[68] G. Gould Phys. Rev., 101(6):1828-1829, 1956.

[69] J.S. Grossman, L.A. Orozco, M.R. Pearson, J.E. Simsarian, G.D. Sprouse and W.Z. Zhao Phys. Rev. Lett., 83(5):935-938, AUG 21999.

[70] Y.P. Gangrsky, B.K. Kuldjanov, K.P. Marinova, B.N. Markov and S.G. Zemlyanoi Z. Phys. D, 42(1):1-4, OCT 1997. 